\title{
Hydrodynamic boundary effects on thermophoresis of confined colloids
}

\author{
Alois Würger \\ Laboratoire Ondes et Matière d'Aquitaine, Université de Bordeaux 8 G CNRS, 33405 Talence, France
}

\begin{abstract}
We study hydrodynamic slowing-down of a particle moving in a temperature gradient perpendicular to a wall. At distances much smaller than the particle radius, $h \ll a$, lubrication approximation leads to the reduced velocity $u / u_{0}=3 \frac{h}{a} \ln \frac{a}{h}$, with respect to the bulk value $u_{0}$. With Brenner's result for confined diffusion, we find that the trapping efficiency, or effective Soret coefficient, increases logarithmically as the particle gets very close to the wall. This provides a quantitative explanation for the recently observed enhancement of thermophoretic trapping at short distances. Our discussion of parallel and perpendicular thermophoresis in a capillary, reveals a very a good agreement with five recent experiments on charged polystyrene particles.
\end{abstract}

PACS numbers:

The motion of a colloid close to a solid boundary is strongly influenced by hydrodynamic interactions. Thus the like-charge attractions observed for confined colloidal assemblies 2, were shown to arise from hydrodynamic fluctuations [1. Similarly, a surface-active particle with a flow field perpendicular to the wall, induces lateral advection of nearby neighbors and cluster formation [3 7]. More recently, the collision patterns observed for selfpropelling Janus particles close to a wall [8], were related to hydrodynamic interactions. Quite generally, the latter are relevant where surface forces and confined geometries are combined for sieving [11, trapping [12, 13], and assembling colloidal beads 14.

A generic example is provided by a surface-active particle moving towards a wall. If hydrodynamic effects on Brownian motion are well understood in terms of Brenner's solution for confined diffusion [15, this is not the case for the drift velocity $u$. At distances $h$ much larger than the particle radius $a$, electrophoresis slows down by the factor $u / u_{0}=1-\frac{5}{8} a^{3} / h^{3}$ [16]. At short distances $h<a$, the wall-solvent-particle permittivity contrast strongly alters the local electric field; this electric coupling is difficult to separate from hydrodynamic interactions; a similar effect influences diffusiophoresis [17. A more favorable situation occurs for thermophoresis, where the drift velocity is proportional to the temperature gradient [18, 19]: Since the heat conductivity of silica or polystyrene (PS) particles is not very different from that of water, the thermal gradient is hardly affected by the presence of the wall [7, and velocity changes can be unambiguously attributed to hydrodynamic interactions.

Here we study the vertical motion of a particle that is confined to the upper half-space $z \geq 0$, as illustrated in Fig. 1; applications are thermophoresis across a capillary and self-propelling Janus particles that preferentially orient toward the wall, or "pullers" [10]. In the steady state, drift and diffusion currents cancel each other, $-u c-D \nabla c=0$, and the particle concentration satisfies

$$
-\nabla \ln c=\frac{u}{D} .
$$

At large distances $h \gg a$, there are no boundary effects

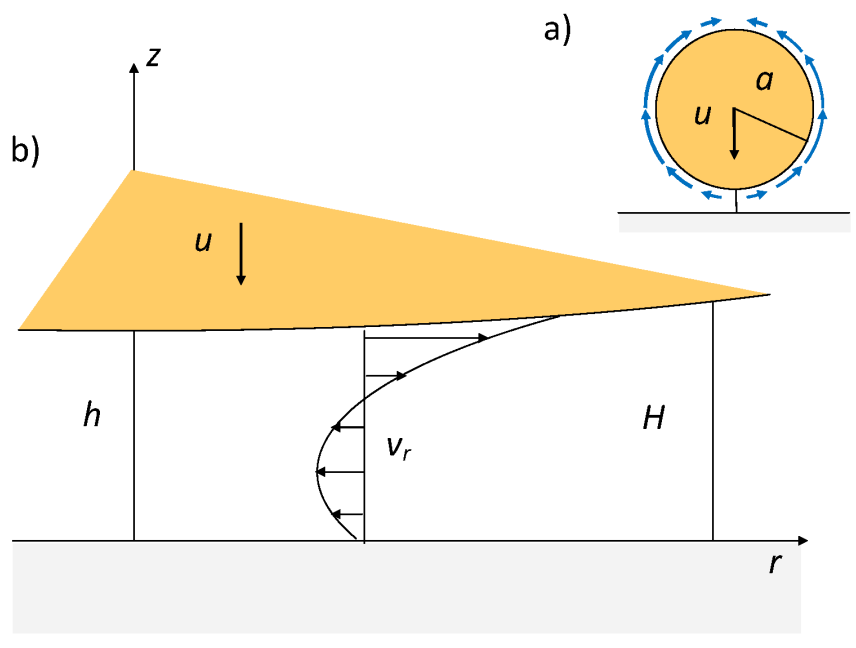

FIG. 1: Schematic view of a particle moving towards a confining wall at velocity $u$. a) The arrows along the particle surface indicate the slip velocity $v_{s}$ induced by thermodynamic forces. b) In the narrow slit of width $H(r)=h+a-\sqrt{a^{2}-r^{2}}$, the vertical particle motion and the outward slip velocity result in an intricate radial flow profile $v_{r}$.

and Eq. (1) is readily integrated, $c=c_{0} e^{-h / \ell_{0}}$, with the trapping length $\ell_{0}=D_{0} / u_{0}$ [20]. As the particle approaches the wall, both drift and diffusion are slowed down by hydrodynamic coupling. As a main result, we calculate the velocity reduction factor $u / u_{0}$ in lubrication approximation $(h<a)$ and by the method of reflections $(h>a)$; with Brenner's expression for the diffusion coefficient [15], we evaluate (1) for both limiting cases.

The present work was partly motivated by the recent observation that thermophoretic trapping at very short distances is much stronger than in the bulk 21. When relating (1) to the Soret coefficient $S_{T}$ through $u / D=$ $S_{T} \nabla T$, our results agree quantitatively with the data and provide strong evidence that the observed increase of $S_{T}$ is of hydrodynamic origin.

Hydrodynamic boundary effects. Particle drift arises from the effective slip velocity $v_{s}$ induced by thermal nonequlibrium properties of the boundary layer [19, 27]; with 
the notation of Fig. 1 one has $v_{s}=\frac{3}{2} u_{0}(r / a)$. As the particle approaches the boundary, the wall squeezes the flow field and thus reduces the drift velocity to the value $u$.

At very short distances, the flow in the slit is well described by lubrication approximation, with the radial velocity field

$$
v_{r}(z)=v_{s}\left(\frac{z}{H}-\frac{3 z(H-z)}{H^{2}}\right)+3 u \frac{r}{H} \frac{z(H-z)}{H^{2}} .
$$

The first term arises from the slip velocity $v_{s}$, and satisfies the conditions $\left.v_{r}\right|_{0}=0$ at the solid boundary and $\left.v_{r}\right|_{H}=v_{s}$ at the particle surface $H=h+a-\sqrt{a^{2}-r^{2}}$. Integrating over $z$, one finds that its net flow vanishes. The second term accounts for the particle velocity $u$; one readily verifies that the vertical volume flow $\pi r^{2} u$ within a radius $r$, is cancelled by the radial flow through a cylinder of radius $r$ and height $H$, that is, the $z$-integral of $2 \pi r v_{r}$.

The relation between the particle velocity $u$ and the surface property $v_{s}$, is established by noting that there is no force $F$ between particle and wall. From the radial component of Stokes' equation, $\partial_{r} P=\eta \partial_{z}^{2} v_{r}$, we obtain the pressure gradient $\partial_{r} P=6 \eta v_{s} / H^{2}-6 \eta u r / H^{3}$ which, upon integration, gives $P(r)$. Performing its surface integral along the wall and using that the diagonal component of the viscous stress vanishes, $\sigma_{z z}=0$, we calculate 22]

$$
F=\int d S P(r, z=0)=0 .
$$

The second equality expresses the fact that there is no mechanical or 'thermophoretic' force acting on the particle.

The condition (3) provides a relation between $u$ and $u_{0}$, and thus quantifies the hydrodynamic effects on the drift velocity,

$$
\frac{u}{u_{0}}=\frac{h}{a} \phi(h / a) \quad(h \ll a),
$$

with

$$
\phi(\hat{h})=3(1+\hat{h}) \frac{\left(2+6 \hat{h}+3 \hat{h}^{2}\right) \ln \frac{\hat{h}+1}{\hat{h}}-\frac{3}{2}(3+2 \hat{h})}{2+9 \hat{h}+6 \hat{h}^{2}-6 \hat{h}(1+\hat{h})^{2} \ln \frac{\hat{h}+1}{\hat{h}}}
$$

and the shorthand notation $\hat{h}=h / a$. For very small distances, $\hat{h} \leq \frac{1}{100}$, this expression simplifies to $\phi=$ $-3(\ln \hat{h}+9 / 4)$. With 44 we have an explicit expression for the velocity profile (2).

Now we turn to the case where the distance exceeds the particle size, $h>a$. Following Keh and Anderson [16, we start from the velocity field in a bulk liquid and evaluate the first reflection at the wall [22. The resulting correction to the particle velocity vanishes as $h^{-3}$,

$$
\frac{u}{u_{0}}=1-\frac{1}{2} \frac{a^{3}}{(h+a)^{3}} \quad(h>a) .
$$

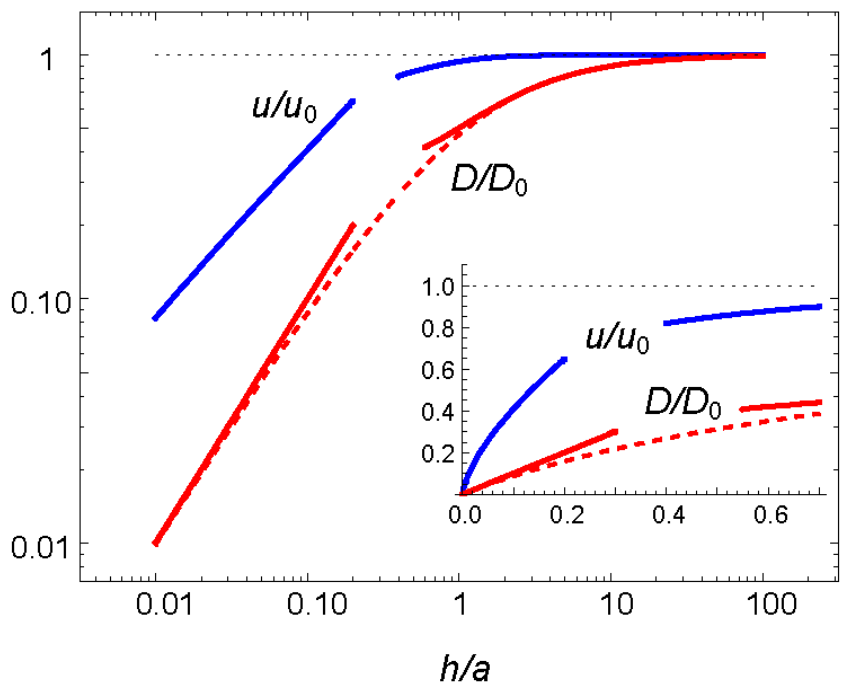

FIG. 2: Reduced drift velocity $u / u_{0}$ and diffusion coefficient $D / D_{0}$ for a particle moving toward a wall, as a function of the relative distance $\hat{h}=h / R$. The curves at small $\hat{h}$ are given by (4) and (6), those at large $\hat{h}$ by (7) and (8). The dashed line is calculated from the first hundred terms of Brenner's exact series for $D / D_{0}[15$. At all distances, the diffusion coefficient is more strongly reduced than the drift velocity. The inset shows the same at linear scale, thus highlighting the linear law $D \propto \hat{h}$ and the logarithmic corrections for $u$ at short distances

A slightly larger correction, with a prefactor $\frac{5}{8}$ instead of $\frac{1}{2}$, was found for the electrophoretic mobility [16]. The difference of $\frac{1}{8}$ arises from the deformation of the electric field by the low-permittivity particle and by the conducting wall. In the case of thermophoresis, the corresponding effect on the local temperature gradient is small, because of the relatively weak thermal conductivity contrast at the particle-solvent-wall interfaces [22]. A more complex situation occurs if ion currents are relevant for the slip velocity, e.g., through the Seebeck effect [20, 23] or a permittivity change due to phase separation [24, 25].

Fig. 2 shows the reduced velocity $u / u_{0}$ as a function of distance; it changes rather little for $h>a$, but drops to zero as $h \rightarrow 0$. For comparison we also plot the corresponding expressions $D / D_{0}$ for the diffusion coefficient. At small distances, the lubrication approximation results in the well-known linear variation with $h$,

$$
D / D_{0}=h / a \quad(h<a),
$$

whereas to third order in the inverse distance, the reflection method results in

$$
\frac{D}{D_{0}}=1-\frac{9}{8} \frac{a}{h+a}+\frac{1}{2} \frac{a^{3}}{(h+a)^{3}} \quad(h>a) .
$$

The dashed curve gives Brenner's exact formula [15]. As a general rule, hydrodynamic slowing down is significantly stronger for diffusion, as a consequence of the long-range velocity field accompanying Browian motion. 


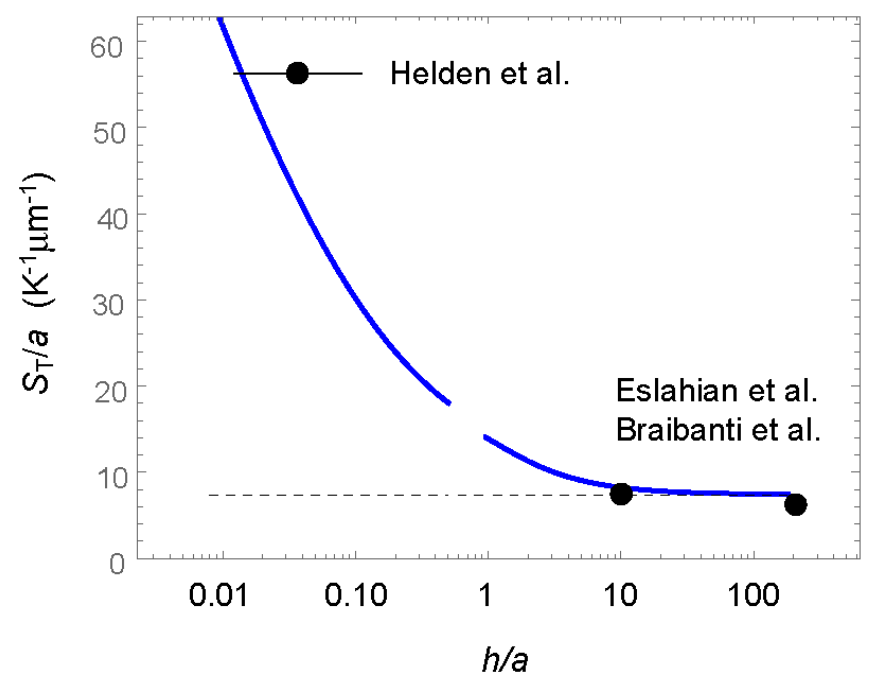

FIG. 3: Hydrodynamic effects on the effective Soret coefficient $S_{T} / a$. The solid lines show the results from lubrication approximation (9) and the method of reflection from 10 ; the dashed line gives the bulk value $S_{T}^{0} / a=7.4 \mathrm{~K}^{-1} \mu \mathrm{m}^{-1}$ [22]. The experimental points are from Table I.

Thermophoretic trapping. In view of a recent Soret experiment by Helden et al. [21, we discuss confined thermophoresis, where the stationary distribution (1) defines the Soret coefficient $S_{T}$ through $u / D=S_{T} \nabla T$. Assuming a constant temperature gradient, one obtains hydrodynamic effects as the ratio of the correction factors for drift and diffusion. In lubrication approximation, this results in

$$
S_{T}=S_{T}^{0} \phi(\hat{h}) \quad(\hat{h}<1),
$$

whereas in the opposite limit we have

$$
S_{T}=S_{T}^{0} \frac{1-\frac{1}{2} \frac{1}{(1+\hat{h})^{3}}}{1-\frac{9}{8} \frac{1}{1+\hat{h}}+\frac{1}{2} \frac{1}{(1+\hat{h})^{3}}} \quad(\hat{h}>1) .
$$

In Fig. 3 we plot the Soret coefficient as a function of the reduced distance $h / a$. As the particle gets closer to the wall, trapping is enhanced by hydrodynamic interactions, the Soret coefficient increases with respect to the bulk value, and at $\hat{h} \rightarrow 0$ diverges logarithmically.

The bulk Soret coefficient.varies linearly with the particle radius, $S_{T}^{0} \propto a$, due to the inverse variation of the Stokes-Einstein coefficient $D_{0}=k_{B} T /(6 \pi \eta a)$ [26] and the constant drift velocity $u_{0}$ [27]. In order to facilitate the comparison of Soret data for particles of different radius, we plot the ratio $S_{T} / a$. Our findings provide a quantitative explanation for the data of Helden et al. [21]: For polystyrene particles $(a=2.5 \mu \mathrm{m})$ very close to a wall $(h<0.3 \mu \mathrm{m})$, these authors reported $S_{T}=140 \mathrm{~K}^{-1}$ at room temperature; the reduced value $S_{T} / a$ is seven times larger than those reported in previous experiments on particles at large distances; see Fig. 3 and Table I. The quantitative agreement with the present theory provides strong evidence that the enhanced trapping is of

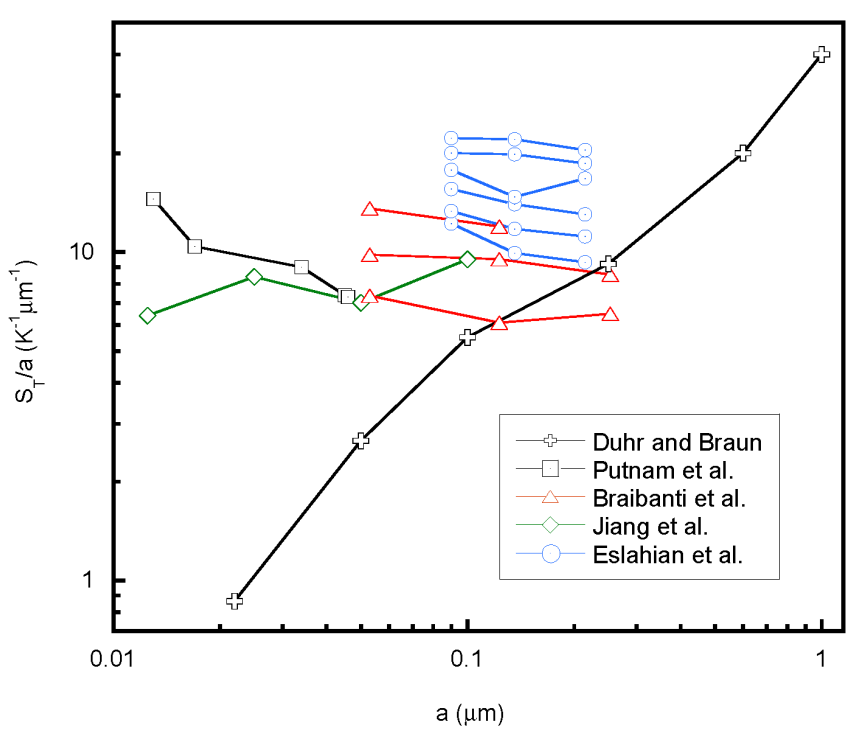

FIG. 4: Size dependence of the Soret coefficient $S_{T}$. For the data of five experiments on polystryene particles, the ratio of $S_{T}$ and the particle radius $a$ is plotted as a function of $a$. The data $(+)$ are taken at room temperature, Duhr and Braun 33]; ( $\square$ ) above $35{ }^{\circ} \mathrm{C}$, Putnam et al. 30; $(\triangle)$ at $25,35,45$ ${ }^{\circ} \mathrm{C}$, Braibanti et al. [31; ; $(\diamond)$ Jiang et al. [32]; $(\bigcirc)$ at 28, 31, $35,39,44,47^{\circ} \mathrm{C}$, Eslahian et al. 20. The lines connect data at constant temperature. Details are given in 22 .

hydrodynamic origin. This is corroborated by the similar temperature series observed at small [21] and large distances [20, 31].

Since the linear size dependence of $S_{T}^{0}$ is essential for the above argument, we recall its theoretical foundation and experimental confirmation. If the Stokes-Einstein coefficient needs no further discussion, a few words are in order concerning $u_{0}$. As first shown by von Smoluchowski in his study of thin-boundary layer electrophoresis [28], the equilibrium between surface forces and viscous stress is independent of the particle radius, and so is the velocity $u_{0}$. Later on, Derjaguin generalized this argument to motion driven by composition and temperature gradients

TABLE I: Soret data for polystyrene particles in capillaries with a perpendicular temperature gradient [20, 21, 31]. The numbers $S_{T} / a$ give the data shown in Fig. 5 [31, or their extrapolation to $T=25^{\circ}[20$; that of Helden et al. is taken from Fig. 4 of 21. The values for the distance $h$ correspond to the range where data are taken [21] or, for weak trapping, to $\ell_{0}=D_{0} / u_{0}$ 20, 31; the value for Ref. 31 indicates a lower bound. For a more detailed analysis, see [22].

\begin{tabular}{|l|c|c|c|}
\hline$T=25^{\circ} \mathrm{C}$ & $\begin{array}{c}S_{T} / a \\
\left(\mathrm{~K}^{-1} \mu \mathrm{m}^{-1}\right)\end{array}$ & $\begin{array}{c}h \\
(\mu \mathrm{m})\end{array}$ & $h / a$ \\
\hline Helden et al. [21] & 56 & $0.03-0.3$ & $0.012-0.12$ \\
\hline Eslahian et al. [20] & 7.4 & 2 & 9 \\
\hline Braibanti et al. 31] & 6.5 & $>50$ & $>200$ \\
\hline
\end{tabular}




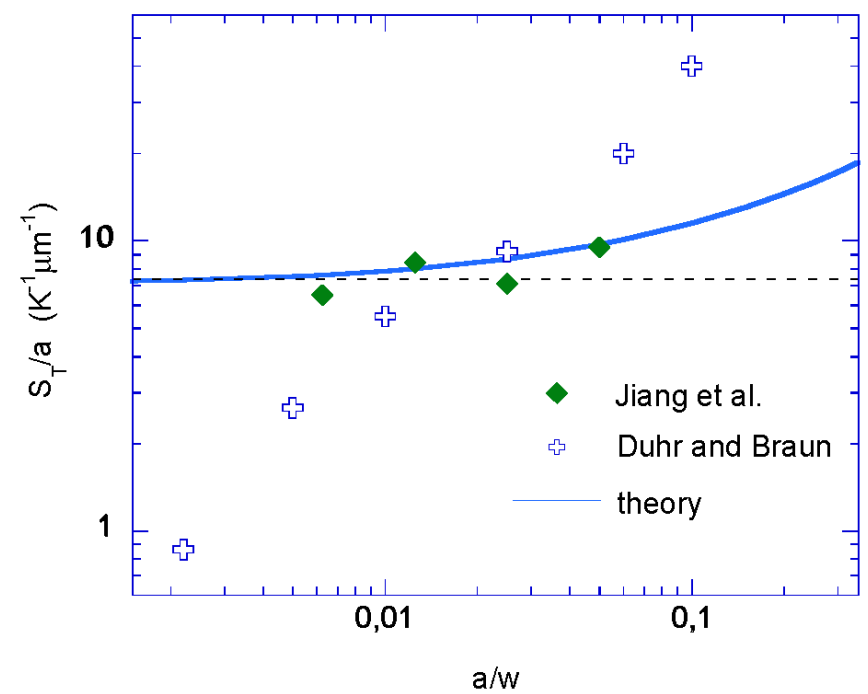

FIG. 5: Thermophoretic trapping parallel to the capillary. We show $S_{T} / a$ as a function of $a / w$. The data of Duhr and Braun are taken in a capillary of width $w=10 \mu \mathrm{m}$ 33, and those of Jiang et al. in $w=2 \mu \mathrm{m}$ [32. The solid line is calculated from 11] where hydrodynamic effects are accounted for by Oseen's model for the parallel diffusion coefficient. The dashed line indicates the bulk value $S_{T}^{0} / a=7.4 \mathrm{~K}^{-1} \mu \mathrm{m}^{-1}$.

[29]. The law $u_{0}=$ const. ceases to be valid for no-stick boundary conditions with large Navier slip length and for particles smaller than the Debye length [19; yet none of these cases is relevant for the systems considered here.

Fig. 4 shows Soret data $S_{T} / a$ as a function of $a$, measured for PS particles in five experiments. In the setup of Refs. [20, 30, 31] the temperature gradient is perpendicular to the boundary as in Fig. 1; a parallel configuration is used in 32, 33, with the particles moving along the capillary. The data of [20, 30 32, show the behavior $S_{T} / a=$ const. expected for large $h$, and even their absolute values agree well with each other. A constant ratio was also observed for surfacted microemulsion droplets 34. On the contrary, Duhr and Braun reported a linear variation $S_{T} / a \propto a$ over two orders of magnitude 33 .

Motion parallel to the capillary. In view of this discrepancy, we complete our discussion of hydrodynamic effects by considering thermophoresis along the boundaries, where confinement is expressed in terms of the ratio of the particle radius $a$ and the width $w$ of the capillary. In view of the experiments [32, 33] we consider the perturbative range $a \ll w$ only. In Oseen's model for parallel diffusion, confinement reduces the Stokes-Einstein coefficient of a particle at vertical position $z$ according to $D_{0} / D_{\|}=1+\frac{9}{8} \frac{a}{z}+\frac{9}{8} \frac{a}{w-z}[15$. The thermophoretic velocity is hardly affected by the walls, in leading order we have $u / u_{0}=1$. Taking the position average in the interval $[a, w-a]$, we find

$$
\frac{S_{T}}{S_{T}^{0}}=1+\frac{9}{4} \frac{a}{(w-2 a)} \ln \frac{w-a}{a} \quad(a \ll w) .
$$

In Fig. 5 we plot the reduced Soret data from Refs. [32, 33. as a function of $a / w$, and compare with the theoretical expression (11). If the four data points of Jiang et al. 32] agree well with theory, this is not the case for those of Duhr and Braun: The Soret effect of the biggest particles $(a=1 \mu \mathrm{m})$ is three times stronger, whereas that of the smallest one $(a=22 \mathrm{~nm})$ is by one order of magnitude too weak. This discrepancy can not be explained by higher-order terms in (11) or by additional effects such as thermoosmosis along the capillary. For a discussion of experimental issues, see Ref. [31.

Conclusion. We have studied hydrodynamc effects on the velocity and the confinement of colloids approaching a wall. At very small distances $\hat{h} \ll 1$, lubrication approximation results in the reduction factor $u / u_{0}=$ $-3 \hat{h} \ln \hat{h}$, which is by a logarithmic factor larger than that of the diffusion coefficient, $D / D_{0}=\hat{h}$.

Our theory provides a quantitative explanation for a recent experiment on confined thermophoresis [21], where the measured Soret coefficient is by almost one order of magnitude larger than expected from previous studies without confinement. This confirms Derjaguin's approach to thermal motion which is based on surface forces and hydrodynamics.

More generally, our findings show that hydrodynamic coupling strongly affects the motion of surface-active colloids in micron-size capillaries or close to solid boundaries.

Stimulating discussions with L. Helden are gratefully acknowledged. This work was supported by Agence Nationale de la Recherche through contract ANR-13-IS040003 ,
[1] T.M. Squires, M.P. Brenner, Phys. Rev. Lett. 85, 4976 (2000)

[2] A.E. Larsen, D. G. Grier, Nature 385, 230 (1997)

[3] M. Trau, D. Saville, and A. I. Askay, Science 272, 706 (1996)

[4] S.R. Yeh, M. Seul, B.I. Shraiman, Nature 386, 57 (1997)

[5] F.M. Weinert, D. Braun, Phys. Rev. Lett. 101, 168301 (2008)

[6] R. Di Leonardo, F. Ianni, G. Ruocco, Langmuir 25, 4247
(2009)

[7] J. Morthomas, A. Würger, Phys. Rev. E 81, 051405 (2010)

[8] G. Volpe, I. Buttinoni, D. Vogt, H.-J. Kümmerer, and C. Bechinger, Soft Matter 7, 8810 (2011)

[9] S.E. Spagnolie and E. Lauga, J. Fluid Mech. 700, 105.(2012)

[10] K. Schaar, A. Zöttl, H. Stark, Phys. Rev. Lett. 115, 038101 (2015) 
[11] A. Cuche, A. Canaguier-Durand, E. Devaux, J. A. Hutchison, C. Genet, T.W. Ebbesen, Nano Lett. 13, 4230 (2013)

[12] J. Chen, Z. Kang, S.K. Kong, and H.-P. Ho, Optics Lett. 40, 3926 (2015)

[13] M. Braun, A. Würger and F. Cichos, Phys. Chem. Chem. Phys. 16, 15207 (2014)

[14] I. Theurkauff, C. Cottin-Bizonne, J. Palacci, C. Ybert, L. Bocquet, Phys. Rev. Lett. 108, 268303 (2012)

[15] H. Brenner, Chem. Eng. Sci. 16, 242 (1961)

[16] H.J. Keh, J.L. Anderson, J. Fluid Mech. 153, 417 (1985)

[17] Y.C. Chang, H.J. Keh, J. Colloid Interf. Sci. 322, 634 (2008)

[18] R. Piazza, Soft Matter 4, 1740 (2008).

[19] A. Würger, Rep. Prog. Phys. 73, 126601 (2010)

[20] K.A. Eslahian, A. Majee, M. Maskos, A. Würger, Soft Matter 10, 1931 (2014)

[21] L. Helden, R. Eichhorn, C. Bechinger, Soft Matter 11, 2379 (2015)

[22] See Supplemental material for technical details

[23] A. Würger, Phys. Rev. Lett. 101, 108302 (2008)
[24] I. Buttinoni, G. Volpe, F. Kümmel, G. Volpe, and C. Bechinger, J. Phys. Condens. Matter 24, 284129 (2012)

[25] A. Würger, Phys. Rev. Lett. 115, 188304 (2015)

[26] A. Einstein, Annal. Phys. 19, 371 (1906)

[27] J.L. Anderson, Ann. Rev. Fluid Mech. 21, 61 (1989)

[28] M. von Smoluchowski, Bull. Int. Acad. Sci. Cracovie 184.(1903)

[29] N.V. Churaev, B.V. Derjaguin, V.M. Muller, Surface Forces, Plenum Publishing Corporation (New York 1987)

[30] S.A. Putnam, D.G. Cahill, G.C.L. Wong, Langmuir 23, 9221 (2007)

[31] M. Braibanti, D. Vigolo, R. Piazza, Phys. Rev. Lett. 100, 108303 (2008).

[32] H.-R. Jiang, H. Wada, N. Yoshinaga and M. Sano, Phys. Rev. Lett. 102, 208301 (2009)

[33] S. Duhr, D. Braun, Phys. Rev. Lett. 97, 168301 (2006)

[34] D. Vigolo, S. Brambilla, R. Piazza, Phys. Rev. E 75, 040401 (2007) 